\title{
Spinal Cord Compression Secondary to Extramedullary Hematopoiesis: Case Report and Review of the Literature
}

\author{
Arthur Wang $^{\mathrm{a}} \quad$ Nathan Carberry $^{\mathrm{a}} \quad$ Elena Solli $^{\mathrm{a}} \quad$ John Gillick $^{\mathrm{a}}$ \\ Humayun Islam $^{\mathrm{b}}$ Virany Hillard ${ }^{\mathrm{a}}$ \\ a Department of Neurosurgery, New York Medical College, Westchester Medical \\ Center, Westchester, N.Y., USA; ${ }^{b}$ Department of Pathology, New York Medical College, \\ Westchester Medical Center, Westchester, N.Y., USA
}

\section{Keywords}

Extramedullary hematopoiesis $\cdot$ Spinal cord compression

\begin{abstract}
Extramedullary hematopoiesis (EMH) is a rare cause of spinal cord compression (SCC). EMH represents the growth of blood cells outside of the bone marrow and occurs in a variety of hematologic illnesses, including various types of anemia and myeloproliferative disorders. Although EMH usually occurs in the liver, spleen, and lymph nodes, it may also occur within the spinal canal. When this occurs, the mass effect can compress the spinal cord, potentially leading to the development of neurological deficits. We present a case of SCC secondary to $\mathrm{EMH}$. This report illustrates the importance of considering EMH in the differential diagnosis of SCC, even in the absence of signs of its most common etiologies.
\end{abstract}




\section{Case Reports in Oncology}

Wang et al.: Spinal Cord Compression Secondary to Extramedullary Hematopoiesis: Case Report and Review of the Literature

\section{Introduction}

Extramedullary hematopoiesis (EMH) is defined as the noncancerous proliferation of hematopoietic tissue outside the bone marrow. The hematologic etiologies vary in pathophysiology, but most of them result in a state of anemia in which there are not enough functional red blood cells to satiate the body's oxygen demands. Conditions that can reduce the amount of functional red blood cells include thalassemia, sickle cell anemia, acquired hemolytic anemia, and vitamin $\mathrm{B}_{12}$ or folate deficiency. Anemia can also be caused by bone marrow radiation used as treatment for some other condition [1-3]. It can also be caused by myelophthisic diseases in which the bone marrow is disrupted, such as leukemia, lymphoma, or myelofibrosis [4]. Congenital hematologic etiologies are some of the more common causes of EMH, and among these thalassemia is particularly suspect [5, 6]. EMH exists as a mechanism to compensate for abnormally low levels of functional red blood cells, but can become pathological if it disrupts the other functions of the body [1, 2].

The most frequent sites of EMH are the liver, spleen, and lymph nodes. Less common sites include the retroperitoneal tissue, kidneys, adrenal glands, paravertebral area, thymus, breasts, sweat glands, pleura, prostate, and broad ligaments [1, 6]. Even less common ectopic locations include peripheral nerves and the cranial dura mater [1].

Very rarely does EMH occur in the spinal canal [2]. The first case of spinal cord compression (SCC) secondary to EMH was reported by Gatto et al. [7] in 1954. Among the documented episodes of thalassemia-associated EMH, intraspinally located growths occur in 11$15 \%$ of cases $[8,9]$. Within the spine, the most common site is the thoracic region, followed by the lumbar region [1]. Importantly, the thoracic spine can be problematic for EMH because the spinal canal is especially narrow in this region $[1,3,10]$. There are several theories that attempt to explain the pathophysiology of EMH within the intraspinal space, including the possibility of direct extension of hematopoietic material from the medulla of the vertebral body [2]. Other models are embolism of hematopoietic tissue into the intraspinal space as well as anomalous activation of embryonic stem cells that reside in this area [11].

If there is a growth in an abnormal location within the body, it follows that naturally residing material might be pathologically displaced via a mass effect. While this does not always result in a loss of function, EMH within the spinal cord can be associated with significant morbidity. One serious complication is SCC [5]. The clinical presentation of SCC secondary to EMH may include back pain, sensory deficits, motor deficits, bowel and bladder dysfunction, and complete paralysis among its symptoms [1,3]. Its severity can range from mild sensory and motor symptoms to total paralysis, and damage can be irreversible if not corrected expeditiously [1]. We present the case of a patient who developed signs and symptoms of EMH-induced SCC, but who lacked any sign of its known etiologies.

\section{Case Report}

Our patient was a 51-year-old female who presented with a 1-week history of lower extremity weakness and mid-thoracic back pain. The patient had a known history of breast cancer diagnosed 10 years ago, and was coded status post mastectomy and radiation. On physical examination, she had $4 / 5$ bilateral lower extremity weakness and diminished sensation below the T4 spinal level.

Magnetic resonance imaging (MRI) revealed a $1.6 \times 1.0 \times 9.7 \mathrm{~cm}$ large enhancing extradural mass in the dorsal aspect of the spinal canal extending from T4 to T11 (fig. 1). There 


\section{Case Reports in Oncology}

Wang et al:: Spinal Cord Compression Secondary to Extramedullary Hematopoiesis: Case Report and Review of the Literature

was near-complete effacement of the spinal cord resulting in SCC. Large bulky enhancing bilateral paraspinal masses were also seen in addition to multiple masses in the lungs and liver that were compatible with metastatic lesions.

Given the patient's neurological deficits and MRI findings, a T4-T8 laminectomy was performed to decompress the spinal cord and resect the lesion. Intraoperatively, the bone was noted to be very soft and the marrow appeared grossly abnormal. The epidural lesion was encased within a capsule, allowing improved resection. The spinal cord itself was significantly compressed and deformed from the mass effect of the lesion. After resection of the lesion, the spinal cord was noted to pulsate, which indicated adequate decompression. An initial frozen section of the bone revealed marrow elements with trilineage hematopoiesis, and a frozen section of the lesion itself revealed a hypercellular lesion.

Microscopic examination revealed the resected mass to be composed of densely cellular hematopoietic marrow showing trilineage hematopoiesis with marked erythroid hyperplasia. Immunohistochemical stains confirmed the presence of all three hematopoietic lineages (fig. 2). There was no evidence of any metastatic tumor, lymphoma, or leukemia.

The patient recovered well from surgery, and her lower extremities returned to full strength several days following surgery. Upon receiving the pathology, the patient was started on adjuvant radiation therapy. A second round of radiation therapy was performed to further shrink the paraspinal masses adjacent to the spinal column. A 3-month follow-up MRI of the spine demonstrated a decompressed spinal cord without recurrence at spinal levels T4-T8 (fig. 3).

\section{Discussion}

The first case of SCC secondary to EMH was reported in 1954, and was managed with surgical decompression and resection excision followed by local radiation [12]. The etiologies of EMH that have been associated with SCC are thalassemia, sickle cell anemia, myelofibrosis, polycythemia vera, acute myeloid leukemia, and myelodysplasia [2]. Among these sources, thalassemia is the most frequent culprit $[5,6]$. However, thalassemia-associated EMH that results in SCC is far from common. In fact, in an article that collected and analyzed all such cases between 1954 and 1992, only 38 were found in the literature [6].

EMH-induced SCC is rare, but the morbidity associated with it is severe. Although most cases of intraspinal EMH are asymptomatic and are discovered incidentally with imaging, significant SCC can occur and result in permanent neurological injury. MRI is now considered to be the diagnostic modality of choice and considered superior to computed tomography (CT). One advantage that MRI has over CT is that it does not involve radiation. Additionally, it can effectively delineate the extent of the EMH mass as well as any involvement of meningeal and epidural tissue $[1,3,13]$. Biopsy is not always required for diagnosis [1], but if needed, it can be obtained via CT-guided needle biopsy [14]. CT imaging of active lesions will demonstrate soft tissue density on CT. Inactive lesions composed of iron deposits will demonstrate high density on CT, whereas fatty deposits will show low density $[3,15]$.

EMH-induced SCC must be diagnosed and treated quickly. Treatment options include surgery, radiotherapy, and hypertransfusion of red blood cells. Often, more than one type of therapy will be utilized for each patient $[1,5]$. As an adjuvant, hydroxyurea is sometimes used for its myelosuppressive properties [1].

When EMH is associated with neurological deficits, surgical intervention is necessary to decompress the neural elements and obtain a tissue diagnosis. However, gross total resec- 
Wang et al.: Spinal Cord Compression Secondary to Extramedullary Hematopoiesis: Case Report and Review of the Literature

tion of an EMH mass is not always possible due to the borderless nature of the hematopoietic process [6]. Surgery also has the associated risks of general anesthesia, which is generally required for spinal operations [13]. Although opinions vary, it is generally agreed that surgical management of EMH should be reserved for acute, severe, or recurrent cases [1, 5]. As our patient presented with the sudden onset of neurological symptoms that did not improve after 2 months, it was appropriate and necessary for the patient to be managed surgically.

For many patients, radiotherapy is emerging as the treatment of choice for EMHinduced SCC because it is of low risk and well tolerated. One group indicated that the EMH volume decreases by $16.4 \%$ immediately after radiotherapy [16]. However, one problem with radiotherapy is that it can exacerbate anemia by decreasing hematopoiesis in the surrounding tissue [1], and therapy cannot be initiated without establishing a diagnosis first [13].

Hypertransfusion of red bloods cells is another rising therapy that is considered to be the least invasive option for the management of EMH-induced SCC [17]. In addition to its therapeutic benefits, hypertransfusion can also be diagnostic, since a response to this treatment indicates an anemic etiology [1]. One problem with this type of treatment is that symptoms can recur shortly afterwards. Hypertransfusion appears to be the best option for mild cases or for diagnostic purposes [17].

Of the cases of EMH-induced SCC within the literature, both the treatments and outcomes have varied widely. For example, one group successfully managed a patient who had chronic thalassemia intermedia with surgical decompression after hypertransfusion had failed to alleviate the neurological symptoms [13]. Another group had immediate success in treating thalassemia intermedia in their patient with hypertransfusion, and the neurological symptoms disappeared within a week [18]. Some other teams utilized hypertransfusion, and then added hydroxyurea for supportive myelosuppression [1, 19].

We present a case of EMH-induced SCC that occurred outside the context of a known hematologic or myeloproliferative disorder. This kind of presentation is rare, but it has previously been documented. One recent case describes a patient presenting with neck pain and stiffness after a minor trauma, which led to the discovery of a neck mass composed of benign hematopoietic tissue. The patient had no known hematologic or myeloproliferative disease [20]. Our patient was similar in that there was no underlying hematologic disease, and the diagnosis was initially suspected to be a new breast cancer metastasis. Given that the treatment for EMH varies dramatically from that for cancer and other more common etiologies of SCC, this case report demonstrates the importance of being mindful of rare diseases.

\section{Statement of Ethics}

The authors have no ethical conflicts to disclose.

\section{Disclosure Statement}

The authors declare that there are no conflicts of interest. 


\section{References}

1 Chehal A, Aoun E, Koussa S, et al: Hypertransfusion: a successful method of treatment in thalassemia intermedia patients with spinal cord compression secondary to extramedullary hematopoiesis. Spine (Phila Pa 1976) 2003;28:E245-E249.

-2 Monti L, Romano D, Gozzetti A, et al: Myelodysplasia presenting as a thoracic spinal epidural extramedullary hematopoiesis: a rare treatable cause of spinal cord myelopathy. Skeletal Radiol 2012;41:611-614.

-3 Haidar R, Mhaidli H, Taher AT: Paraspinal extramedullary hematopoiesis in patients with thalassemia intermedia. Eur Spine J 2010;19:871-878.

-4 Orphanidou-Vlachou E, Tziakouri-Shiakalli C, Georgiades CS: Extramedullary hemopoiesis. Semin Ultrasound CT MR 2014;35:255-262.

-5 Martínez-Rodrigo MA, Sanjuanbenito L, Rodríguez del Barrio E, et al: Spinal cord compression secondary to epidural extramedullary hematopoiesis in thalassemia: a clinical case and review of literature (in Spanish). Rev Neurol 1998;27:998-1004.

6 Singhal S, Sharma S, Dixit S, et al: The role of radiation therapy in the management of spinal cord compression due to extramedullary haematopoiesis in thalassemia. J Neurol Neurosurg Psychiatry 1992;55:310-312.

7 Gatto I, Terrana V, Biondi L: Compression of the spinal cord due to proliferation of bone marrow in epidural space in a splenectomized person with Cooley's disease. Haematologica 1954;38:61-76.

-8 Amirjamshidi A, Abbassioun K, Ketabchi SE, et al: Spinal extramedullary hematopoiesis in adolescents with thalassemia: report of two cases and a review of the literature. Childs Nerv Syst 1991;7:223-225.

9 Dore F, Cianciulli R, Rovasio S, et al: Incidence and clinical study of ectopic erythropoiesis in adult patients with thalassemia intermedia. Ann Ital Med Int 1992;7:137-140.

10 Luitjes WF, Braakman R, Abels J: Spinal cord compression in a new homozygous variant of betathalassemia. Case report. J Neurosurg 1982;57:846-848.

11 Cardia E, Toscano S, La Rosa G, et al: Spinal cord compression in homozygous beta-thalassemia intermedia. Pediatr Neurosurg 1994;20:186-189.

12 Jackson DV Jr, Randall ME, Richards F 2nd: Spinal cord compression due to extramedullary hematopoiesis in thalassemia: long-term follow-up after radiotherapy. Surg Neurol 1988;29:389-392.

13 Coșkun E, Keskin A, Süzer T, et al: Spinal cord compression secondary to extramedullary hematopoiesis in thalassemia intermedia. Eur Spine J 1998;7:501-504.

14 Sproat IA, Dobranowski, Chen V, et al: Presacral extramedullary hematopoiesis in thalassemia intermedia. Can Assoc Radiol J 1991;42:278-282.

15 Tsitouridis J, Stamos S, Hassapopoulou E, et al: Extramedullary paraspinal hematopoiesis in thalassemia: CT and MRI evaluation. Eur J Radiol 1999;30:33-38.

16 Cianciulli P, Sorrentino F, Morino L, et al: Radiotherapy combined with erythropoietin for the treatment of extramedullary hematopoiesis in an alloimmunized patient with thalassemia intermedia. Ann Hematol 1996;72:379-381.

17 Evrard C, Pichereau D, Alsweis S, et al: Extramedullary hematopoiesis of thoracic and vertebral intraductal localization. A propos of a case. Review of the literature (in French). Ann Chir 1994;48:284293.

18 Lee AC, Chiu W, Tai KS, et al: Hypertransfusion for spinal cord compression secondary to extramedullary hematopoiesis. Pediatr Hematol Oncol 1996;13:89-94.

19 Bruneteau G, Fénelon G, Khalil A, et al: Spinal cord compression secondary to extramedullary hematopoiesis in a patient with thalassemia (in French). Rev Neurol (Paris) 2000;156:510-513.

20 Mattei TA, Higgins M, Joseph F, et al: Ectopic extramedullary hematopoiesis: evaluation and treatment of a rare and benign paraspinal/epidural tumor. J Neurosurg Spine 2013;18:236-242. 


\section{Case Reports in Oncology}

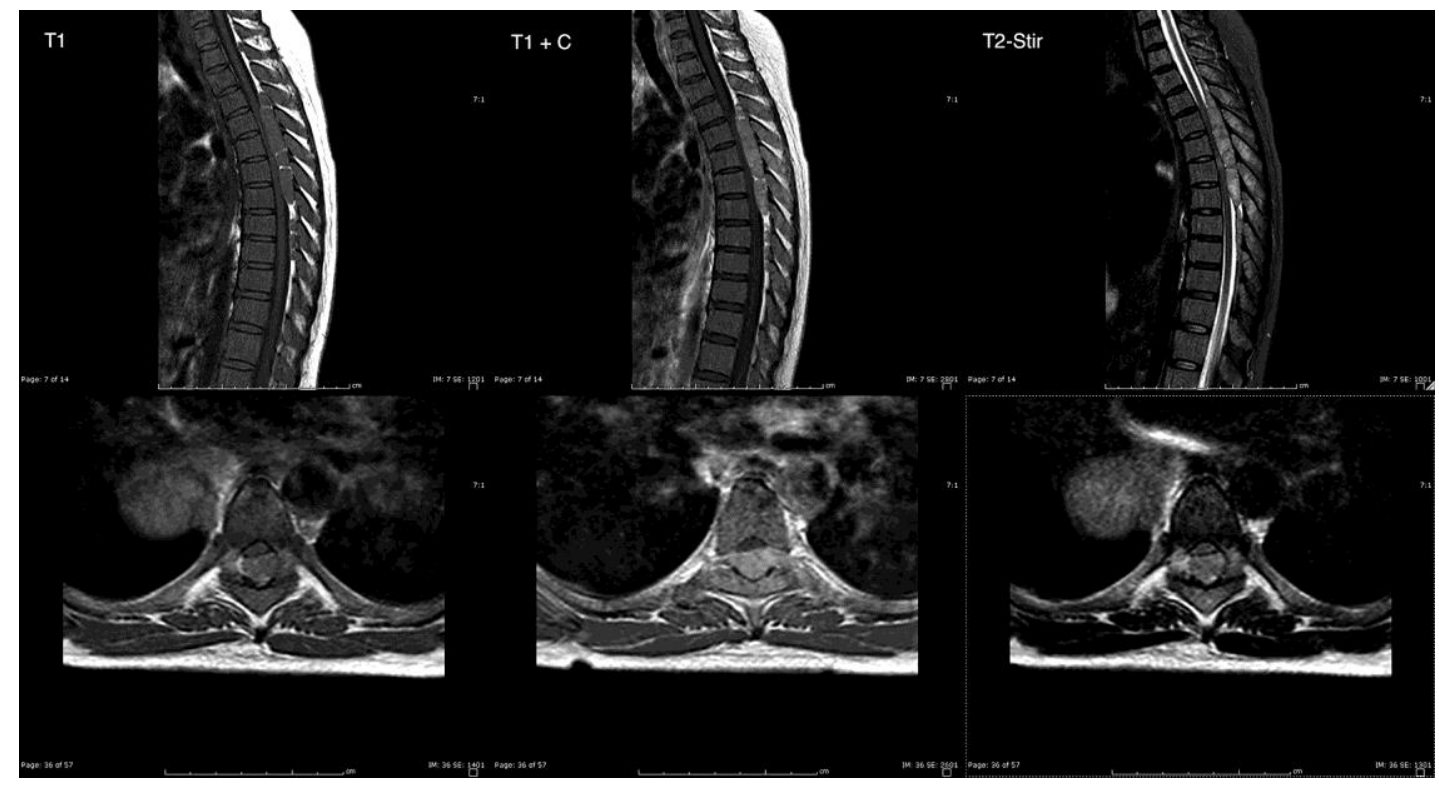

Fig. 1. Sagittal (upper panels) and axial (lower panels) T1-weighted, T1-weighted with Gd (T1 + C), and T2weighted fat suppression (T2-Stir) sequences showing a large enhancing extradural mass in the dorsal aspect of the spinal canal with near-complete effacement of the spinal cord resulting in SCC. 


\section{Case Reports in Oncology}

\begin{tabular}{l|l}
\hline Case Rep Oncol 2016;9:290-297 \\
\hline $10.1159 / 000446473$ & $\begin{array}{l}\text { C 2016 The Author(s). Published by S. Karger AG, Basel } \\
\text { www.karger.com/cro }\end{array}$ \\
\hline
\end{tabular}

Wang et al:: Spinal Cord Compression Secondary to Extramedullary Hematopoiesis: Case Report and Review of the Literature
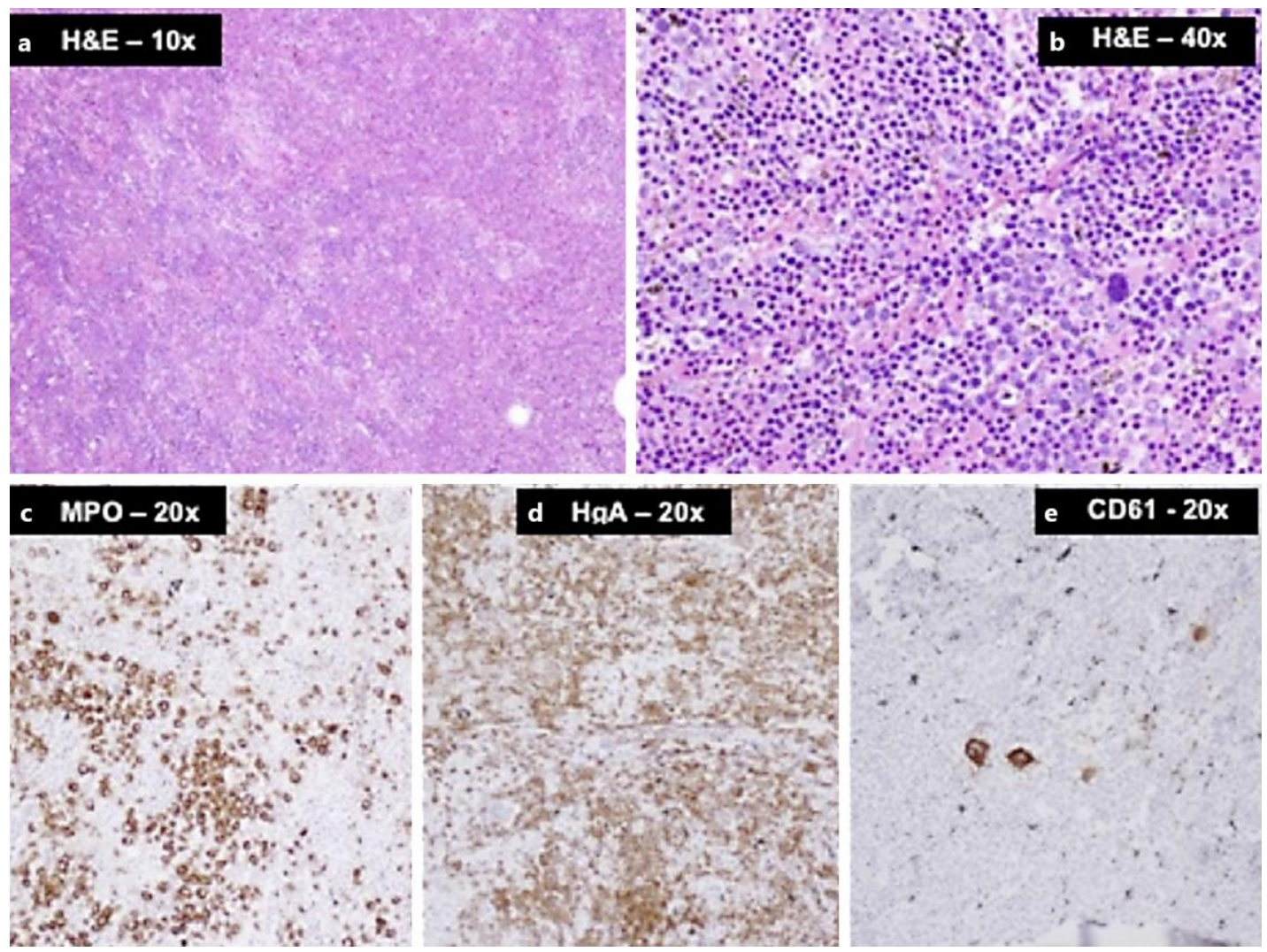

Fig. 2. a, b Photomicrographs of tumor sections with H\&E staining showing densely cellular hematopoietic marrow composed of hyperplastic erythroid elements admixed with myeloid cells and scattered megakaryocytes (large cells). c-e Immunohistochemical stains for myeloperoxidase (MPO), hemoglobin A (HgA), and CD61 highlighting the three lineages, i.e. myeloid, erythroid, and megakaryocytic cells, respectively. 


\section{Case Reports in Oncology}

\begin{tabular}{l|l}
\hline Case Rep Oncol 2016;9:290-297 \\
\hline $10.1159 / 000446473$ & $\begin{array}{l}\text { C 2016 The Author(s). Published by S. Karger AG, Basel } \\
\text { www.karger.com/cro }\end{array}$ \\
\hline
\end{tabular}

Wang et al.: Spinal Cord Compression Secondary to Extramedullary Hematopoiesis: Case Report and Review of the Literature
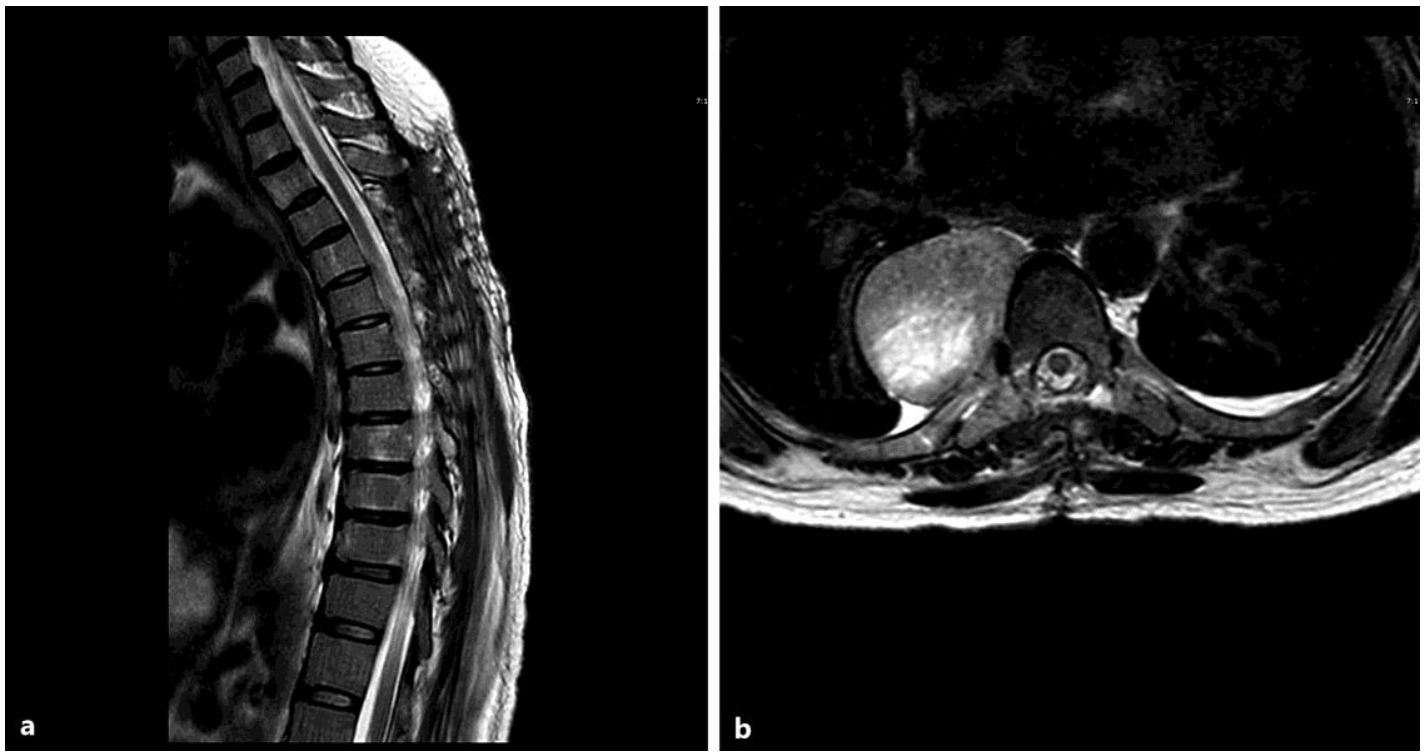

Fig. 3. Sagittal (a) and axial (b) T2-weighted MRI sequences demonstrating the decompressed thoracic spinal cord with cerebrospinal fluid surrounding the cord without local tumor recurrence at the levels operated on. 